\title{
STRUCTURE FORMATION IN DYNAMICAL DARK ENERGY MODELS
}

\author{
A.V. Maccio' ${ }^{1}$, S.A. Bonometto ${ }^{1}$, R, Mainini ${ }^{1} \&$ A. Klypin ${ }^{2}$ \\ (1) Physics Dep. G. Occhialini, Univ. of Milano-Bicocca \& I.N.F.N., Sezione di Milano \\ Piazza della Scienza 2, 20126 Milano, Italy \\ (2) New Mexico State University, Las Cruces, New Mexico, USA \\ maccio@mib.infn.it, bonometto@mib.infn.it, mainini@mib.infn.it, aklypin@nmsu.edu
}

\begin{abstract}
We perform n-body simulations for models with a DE component. Besides of DE with constant negative $w=p / \rho \geq-1$, we consider DE due to scalar fields, self-interacting through RP or SUGRA potentials. According to our post-linear analysis, at $z=0$, DM power spectra and halo mass functions do not depend on DE nature. This is welcome, as $\Lambda \mathrm{CDM}$ fits observations. Halo profiles, instead, are denser than $\Lambda \mathrm{CDM}$. For example, the density at $10 h^{-1} \mathrm{kpc}$ of a DE $\sim 10^{13} M_{\odot}$ halo exceeds $\Lambda$ CDM by $\sim 40 \%$. Differences, therefore, are small but, however, DE does not ease the problem with cuspy DM profiles. On the contrary it could ease the discrepancy between $\Lambda \mathrm{CDM}$ and strong lensing data (Bertelmann 1998, 2002). We study also subhalos and find that, at $z=0$, the number of satellites coincides in all DE models. At higher $z$, DE models show increasing differences from $\Lambda \mathrm{CDM}$ and among themselves; this is the obvious pattern to distinguish between different $\mathrm{DE}$ state equations.
\end{abstract}

Keywords: Audio quality measurements, perceptual measurement techniques

\section{Introduction}

Deep survey and CBR data confirm that $\sim 70 \%$ of the world is Dark Energy (see, e.g., Efstathiou et al 2002, Percival et al 2002, Spergel et al 2003, Tegmark et al 2001, Netterfield et al 2002, Pogosian et al 2003, Kogut et al 2003), as needed to have the accelerated expansion shown by SNIa data (Riess et al 1998, Perlmutter et al 1999). The nature of Dark Energy (DE) is a puzzle. $\Lambda \mathrm{CDM}$ needs a severe fine-tuning of vacuum energy. DE with constant negative $w=p / \rho>-1$ has even less physical motivation. Apparently, the only viable alternative is dynamical DE, a classical self-interacting scalar field $\phi$ (Wetterich 1985). Among potentials $V(\phi)$ with a tracker solution, limiting the impact of initial conditions, Ratra-Peebles (1988, RP hereafter) and SUGRA (Brax \& Martin 1999, 2000) potentials bear a particle physics motivation. 


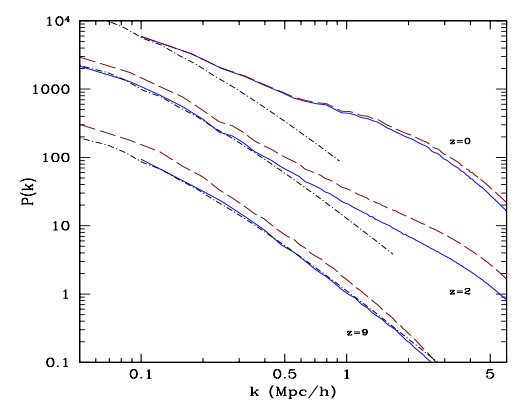

Figure 1. Spectrum evolution for $\Lambda \mathrm{CDM}$ (solid line) and RP (long dashed); the dot-dashed line is the linear prediction for $\Lambda$ CDM.

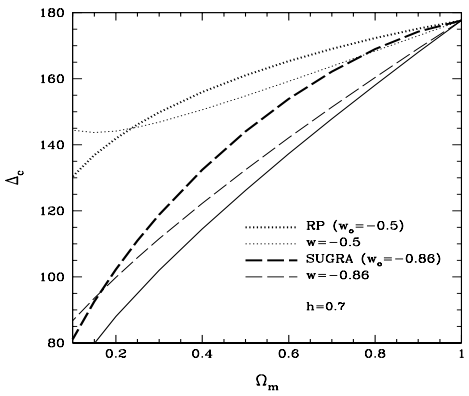

Figure 2. The virial density contrast $\Delta_{c}$ vs. $\Omega_{m}$ at $z=0$. Models are indicated in the frame

Studying a dynamical DE model requires: (i) a linear treatment, to yield CBR spectra and transfer function; (ii) a post-linear treatment, to yield halo virial density contrasts and mass functions; (iii) a non-linear treatment. Here we report results on (ii) and (iii). We use the $\mathrm{n}$-body program ART, modified to deal with any dependence of $\Omega_{m}$ (matter density parameter) on $a$ (scale factor). Mainini et al (2003b) give analytical fitting formulae for such dependence. Further details are in Mainini, Maccio' \& Bonometto (2003a) and Klypin et al (2003). RP and SUGRA are parametrized by the energy scale $\Lambda / \mathrm{GeV}$.

\section{Non-linear results}

Fig. 1 shows the evolution of the spectrum, as obtained from simulations of $\Lambda \mathrm{CDM}$ and RP models $\left(\Lambda / \mathrm{GeV}=10^{3}\right)$, the most distant models treated. Models were normalized so to obtain the same number of halos at $z=0$.

Halos were extracted from simulations using the virial density contrasts $\Delta_{c}$ obtained by Mainini et al (2003b), where one can find plots for the dependence $\Delta_{c}(a)$; here we show $\Delta_{c}$ dependence on $\Omega_{m}$ at $z=0$ (Fig. 2) Figs. $3 \& 4$ show the mass function and its evolution in a number of models.

Using ART facilities, a particular halo was magnified in all simulations. Fig. 5 shows that its profile is NFW with a concentration depending on DE nature. Concentration can also be considered on a statistical basis. Fig. 6 shows how halo concentrations depend on the model. Here concentrations are defined as the ratio between the radius $r_{c}$ at which the density contrast is 110 and the radius $r_{s}$ in the NFW expression of the radial density.

We also studied how the number of satellites of a halo depends on DE nature. In Fig. 7 we report such dependence. However, also in this case, once care is 


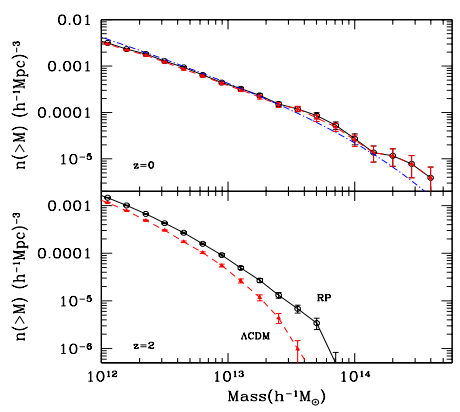

Figure 3. Mass function at $z=0$ and $z=2$ for the same models of Fig. 1. Evolution is faster for $\Lambda \mathrm{CDM}$ than for RP

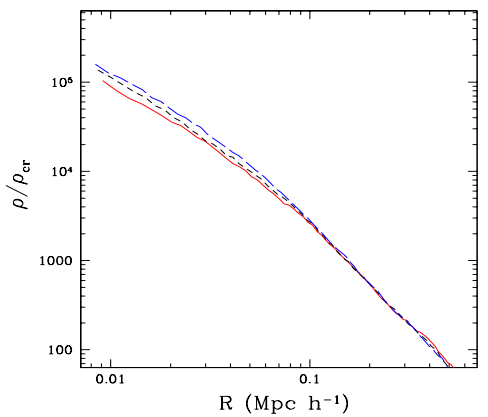

Figure 5. Density profile for a single magnified halo. Solid, short dashed, long dashed lines refer to $\Lambda$ CDM, SUGRA, RP.

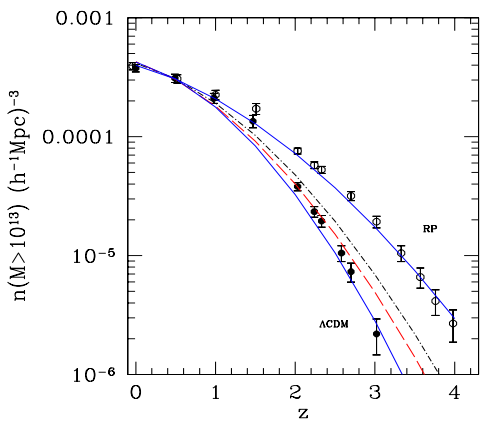

Figure 4. Halo number evolution in various models. Unlabeled curves refer to SUGRA and constant $w=-0.8$

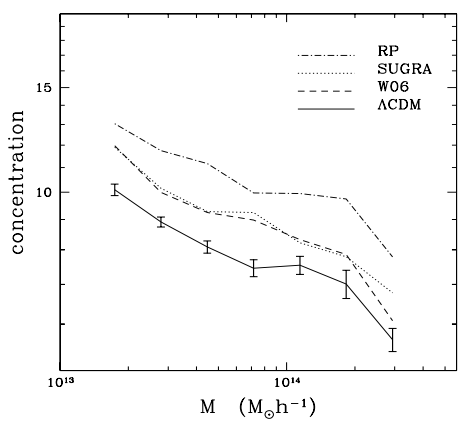

Figure 6. Concentration distribution in various models

payed to properly normalize numbers to the same central halo velocity, no appreciable dependence on DE nature can be found.

\section{Conclusions}

In this paper we showed how a simple modification of the program ART permits to perform a wide analysis of dynamical DE models. This task is simplified by the very structure of the program, which uses the scale factor $a$ as time-variable and requires only

$$
d t / d a=H_{o}^{-1} \sqrt{a \Omega_{m}(a) / \Omega_{m}\left(a_{o}\right)}
$$

( $H_{o}$ : today's Hubble parameter), to detail the action of forces. Once $\Omega_{m}(a)$ (the dependence of the matter density parameter on the scale factor) is as- 


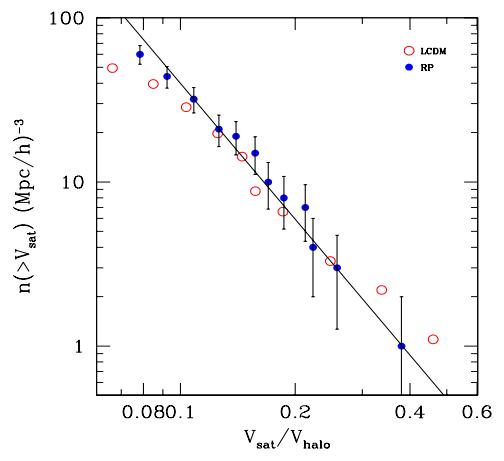

Figure 7. Number of halo satellites

signed, the dynamical problem is then properly defined. Most of the preliminary work was then performed at the post-linear level. This provided us suitable expressions for the virial density contrast, so that halos can be selected in the correct way in all models, and also the required fitting expressions for $\Omega_{m}(a)$.

Discriminating DE models from $\Lambda \mathrm{CDM}$ essentially requires good data at high redshift. A discrimination at $z=0$ can be made only using an observable sensitive to the concentration distribution. In principle, such an observable exists and is related to strong lensing (giant halo statistic). Further work in this direction is in progress.

\section{References}

Bartelmann M., Huss A., Carlberg J., Jenkins A. \& Pearce F. 1998, A\&A 330, 1

Bartelmann M., Perrotta F. \& Baccigalupi C. 2002, A\&A 396, 21

Brax, P. \& Martin, J., 1999, Phys.Lett., B468, 40 and 2000, Phys.Rev. D, 61, 103502

Efstathiou, G. et al., 2002, MNRAS, 330, 29

Klypin, A., Maccio' A.V., Mainini R. \& Bonometto S.A., 2003, ApJ in press, astro-ph/0303304

Kogut et al., 2003, astro-ph/0302213

Mainini R., Maccio’ A.V. \& Bonometto S.A., 2003a, NewA 8, 172

Mainini R., Maccio’ A.V., Bonometto S.A., \& Klypin, A., 2003b, ApJ in press, astro-ph/0303303

Netterfield, C. B. et al. 2002, ApJ, 571, 604

Percival W.J. et al., 2002, astro-ph/0206256, MNRAS (in press)

Perlmutter S. et al., 1999, ApJ, 517, 565

Pogosian, D., Bond, J.R., \& Contaldi, C. 2003, astro-ph/0301310

Ratra B., Peebles P.J.E., 1988, Phys.Rev.D, 37, 3406

Riess, A.G. et al., 1998, AJ, 116, 1009

Spergel et al. 2003, astro-ph/0302209

Tegmark, M., Zaldarriaga, M., \& Hamilton, AJ 2001, Phys.R., D63, 43007

Wetterich C., 1985, Nucl.Phys.B, 302, 668 